Article

\title{
Guanidine Affects Differentially the Twitch Response of Diaphragm, Extensor Digitorum Longus and Soleus Nerve-Muscle Preparations of Mice
}

\section{Rosana Ferrari $^{1,2}$, Léa Rodrigues-Simioni ${ }^{3}$ and Maria Alice da Cruz Höfling ${ }^{1, *}$}

1 Department of Histology and Embryology, Institute of Biology, University of Campinas (UNICAMP), P.O. Box 6109, 13 083-970, Campinas, SP, Brazil; E-Mail: ro.ferrari74@hotmail.com

2 Department of Biology, Institute of Biosciences, São Paulo State University (UNESP), Av. 24A, no.1515, Bela Vista, CEP 13506-900, Rio Claro, São Paulo, Brazil

3 Department of Pharmacology, Faculty of Medical Sciences, University of Campinas (UNICAMP), P.O. Box 6111, 13 083-970, Campinas, SP, Brazil; E-Mail: simioni@unicamp.br

* Author to whom correspondence should be addressed; E-Mail: hofling@unicamp.br; Tel.: +55-19-3521-6224; Fax: +55-19-3289-3124.

Received: 22 May 2012; in revised form: 6 June 2012 / Accepted: 7 June 2012 /

Published: 15 June 2012

\begin{abstract}
Guanidine has been used with some success to treat myasthenia gravis and myasthenic syndrome because it increases acetylcholine release at nerve terminals through $\mathrm{K}^{+}, \mathrm{Na}^{+}$and $\mathrm{Ca}^{2+}$ channels-involving mechanisms. Currently, guanidine derivatives have been proposed for treatment of several diseases. Studies aimed at providing new insights to the drug are relevant. Experimentally, guanidine $(10 \mathrm{mM})$ induces on mouse phrenic nerve-diaphragm (PND) preparations neurotransmission facilitation followed by blockade and a greatest secondary facilitation after its removal from bath. Herein, we hypothesized that this peculiar triphasic response may differ in muscles with distinct twitch/metabolic characteristics. Morphological alterations and contractile response of PND, extensor digitorum longus (EDL) and soleus (SOL) preparations incubated with guanidine (10 $\mathrm{mM})$ for 15, 30, $60 \mathrm{~min}$ were analyzed. Guanidine concentrations of $5 \mathrm{mM}$ (for PND and EDL) and $1 \mathrm{mM}$ (for EDL) were also tested. Guanidine triphasic effect was only observed on PND regardless the concentration. The morphological alterations in muscle tissue varied along time but did not impede the PND post-wash facilitation. Higher doses (20-25 mM) did not increase EDL or SOL neurotransmission. The data suggest a complex mechanism likely dependent on the metabolic/contractile muscle phenotype; muscle fiber types and density/type of ion channels, sarcoplasmic reticulum and mitochondria organization may
\end{abstract}


have profound impact on the levels and isoform expression pattern of $\mathrm{Ca}^{2+}$ regulatory membrane proteins so reflecting regulation of calcium handling and contractile response in different types of muscle.

Keywords: neuromuscular transmission; transmitter release; contractility; skeletal muscle

\section{Introduction}

The contractile response of skeletal muscle can be affected by a variety of substances which can act directly either on the neurotransmission or contractile apparatus, inhibiting and/or facilitating the muscular response. Some of these substances are from animal [1-4] or plant origin [5-7]; others are synthetic products such as tetraethylammonium, aminopyridines and guanidine. All of these substances have been used as tools to improve our understanding on the mechanisms of functioning of neuromuscular junction (NMJ). The highly specific action of these substances allowed them to mimic physiological and pathophysiological events related to muscle contractility, advancing in the knowledge of ion channels and contractile machinery function/dysfunction during excitationcontraction (EC) coupling. For instance, tetraethylammonium (TEA) or 3,4-diaminopyridine $(3,4-\mathrm{DAP})$ increases action potential duration in nerve terminals through the blockade of $\mathrm{K}^{+}$channels, resulting in increased $\mathrm{Ca}^{2+}$ influx into the terminal and release of acetylcholine (ACh) [8-10]. The prolongation of the presynaptic action potential caused by $\mathrm{K}^{+}$channel blockade and further activation of voltage-dependent $\mathrm{Ca}^{2+}$ channels at nerve terminals are also properties displayed by guanidine [11]. TEA, 3,4-DAP and guanidine antagonize the neuromuscular paralysis produced by botulinum toxin [11]. The guanidine molecule $\left[\mathrm{HN}=\mathrm{C}\left(\mathrm{NH}_{2}\right)_{2}\right]$ can also reverse the neuromuscular blocking effect of dibekacin and d-tubocurarine $[12,13]$. The intensity of these effects has been shown to be influenced by $\mathrm{Ca}^{2+}$ intracellular concentration and would result mainly from blockade of fast $\mathrm{K}^{+}$channels, and then modulation of $\mathrm{Ca}^{2+}$ entry into nerve terminals [14]. In the past, guanidine was used with some success to treat myasthenia gravis [15] and myasthenic syndrome [16] because of its ability to improve the neuromuscular transmission, as first described by Feng [17,18]. Guanidine was also shown to be protective against gramicidin toxicity in NG108-15 (neuroblastoma x glioma) hybrid cells [19]. Recently, guanidine derivatives have been proposed for therapeutic use in neuromuscular disorders [20], indicating the drug potential as a research tool in neurobiology. Experimentally, we have reproduced the facilitation of ACh release by guanidine in the chick biventer cervicis and mouse phrenic nerve-diaphragm (PND) preparations; interestingly, a peculiar triphasic effect characterized by an initial facilitation followed by a total neuromuscular blockade and then a secondary facilitation, with amplitude much greater than the former, was evoked when guanidine $(10 \mathrm{mM})$ was removed from bath by washings [21]. In addition, studies with light and transmission electron microscopy showed that a number of fibers showed swelling and rupture of mitochondria and sarcoplasmic reticulum (SR), and alterations in pre- and postsynaptic structures of the NMJ [22]. Other fibers remained morphologically normal. These changes are compatible with ionic unbalance and in line with the inhibitory effect of guanidine on $\mathrm{K}^{+}$and $\mathrm{Ca}^{2+}$ channels and $\mathrm{Na}^{+}$conductance. Such morphological disturbances were only partially prevented by tetrodotoxin (TTX) preincubation. Since the triphasic effect of guanidine on 
PND was fully accomplished after 60 min of incubation [21], it is clear that the alterations produced on mitochondria, SR and pre- and postsynaptic components [22] did not prevent the EC coupling machinery to promote the post-washing marked secondary facilitation. Whether the triphasic effect could occur in earliest periods of incubation, such as 15 and $30 \mathrm{~min}$, corresponding to primary facilitation and reduction phase of the contractile response, respectively, has not been investigated so far. Likewise, the proportion of morphological changes at these earliest periods when compared with those observed after $60 \mathrm{~min}$ of incubation is unknown. Would the extension of damage have correlation with the contractile response? In addition, could the triphasic effect and proportion of muscle damage seen in the mouse diaphragm be equally evoked by guanidine in peripheral (limb) muscle preparations?

The diaphragm is a muscle with distinctive physiological properties when compared with peripheral muscles; it is resistant to the blocking effects of competitive neuromuscular blocking agents due to the high concentration of quanta of $\mathrm{ACh}$ released after stimulation and greater number of occupied postsynaptic nicotinic receptors [23]; along with a low acetylcholinesterase activity at the synaptic cleft [24] and a major quantal content when compared, for example, with EDL [23]. Moreover, the diaphragm muscle comprises motor units varying in their mechanical and fatigue properties and which rely on the fiber type motor innervation control [25]. Interestingly, the decline of quantal content was shown to vary across NMJs of different muscle fiber types during repetitive stimulation, indicating that the underlying mechanism of synaptic delivery is in line with their contractile characteristics [26]. Since fast and slow twitch muscle fibers have distinct contractile properties (see [27] for review), it is not surprising that muscles with different proportion of these fiber types may respond differently to substances affecting neurotransmission.

Herein, we hypothesized that the neuromuscular response to guanidine may differ in muscles with distinct twitch/metabolic characteristics, i.e., the proportion of fibers with predominance of fast-twitch and low-twitch fibers and glycolytic and oxidative metabolism. The contractile response and morphology of phrenic nerve-diaphragm (PND), extensor digitorum longus (EDL) and soleus (SOL) preparations were investigated for 15, 30 and $60 \mathrm{~min}$ of guanidine incubation. The discussion of the results considered the characteristics of each muscle and the pharmacological/ultrastructural effects already described for guanidine on diaphragm after $60 \mathrm{~min}$ incubation. The elucidation of these issues will contribute to shed light on the guanidine pharmacological interaction with phenotypically different skeletal muscles and hence their neurotransmission mechanisms. A variety of pathophysiological disturbances are related to the dysfunction of $\mathrm{K}^{+}$channel and $\mathrm{Na}^{+}$channel activity and calcium homeostasis [28,29]. The use of depolarizing drugs affecting $\mathrm{K}^{+}, \mathrm{Na}^{+}$and $\mathrm{Ca}^{2+}$ channels-dependent cellular events can be excellent tools for determining cell mechanisms, which results in either therapeutic benefits or undesirable side-effects.

\section{Results and Discussion}

\subsection{Myographic Recording}

Myographic recordings of PND, EDL and SOL preparations incubated with guanidine for 15, 30 and $60 \mathrm{~min}$, respectively are shown in Figures 1-3. In PND preparation, guanidine (10 $\mathrm{mM})$ 
determined a gradual neurotransmission facilitation which achieved a $35 \%$ increase after 15 min $(P \leq 0.05)$. The removal of guanidine from bath led to a second phase of facilitation of the contractile response, which was immediate rather than gradual, and almost four times $(\sim 170 \%)$ the amplitude of the initial record. Twenty minutes after guanidine removal by washing the preparation, the increase in the contractile response still remained $150 \%$ above that observed before guanidine administration and in relation to that presented by preparations incubated with Tyrode only (Figure 1A,B).

Figure 1. Effect of guanidine $(10 \mathrm{mM})$ (white circles) for $15 \mathrm{~min}$ on the twitch response of PND (A), EDL (C) and SOL (E) (n=8/each) in comparison with matched controls (black circles) ( $\mathrm{n}=3,8,8$, respectively). The line breaking corresponds to the preparation washing $(\mathrm{W})$. Each point represents the mean \pm S.E.M; one-way ANOVA followed by Tukey test, $* P \leq 0.05$. Panels B (PND), D (EDL) and F (SOL) display the myographic isometric twitch record of preparations incubated with guanidine $(\mathrm{G})$. The vertical bars $(5,0.5 \mathrm{~g})$ represent muscle tension.

(A)

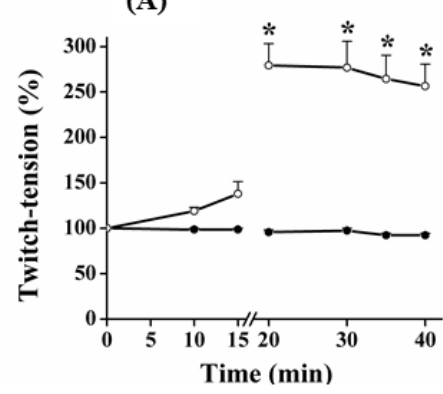

(C)

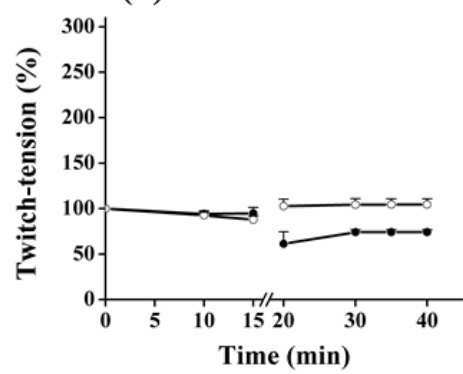

(E)

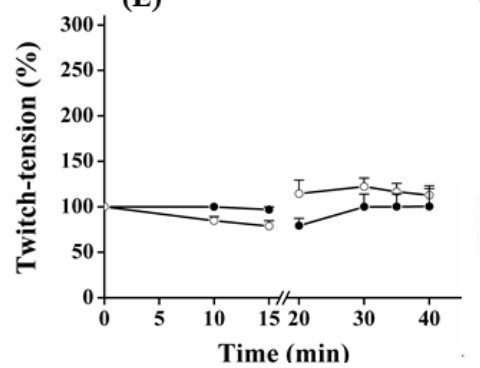

(B)

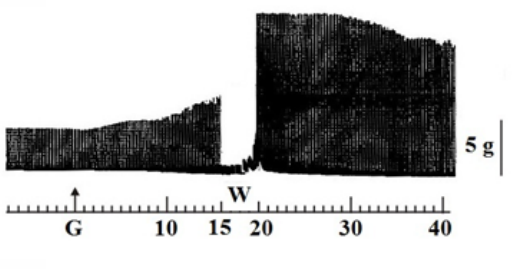

(D)

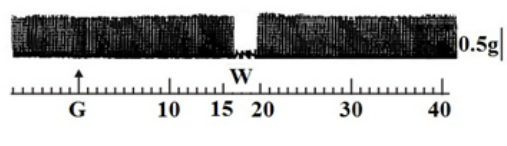

(F)

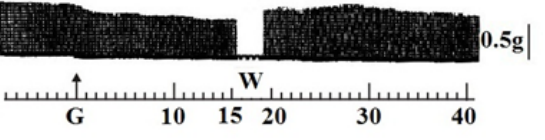

Guanidine $(10 \mathrm{mM})$ did not promote facilitation of neurotransmission in EDL and SOL preparations after 15 min of incubation (Figure 1C,D and 1E,F, respectively). On the contrary, both tended to have reduced responses, which was more evident in SOL. Guanidine washing caused a nonsignificant tendency to increase the contractile response up to the remaining $20 \mathrm{~min}$ of observation. Likewise, neither $1 \mathrm{mM}$ nor $5 \mathrm{mM}$ guanidine promoted facilitation in EDL preparation even after its removal by washing (not shown). 
PND contractile response, after the addition of guanidine $(10 \mathrm{mM})$ in the bath for $30 \mathrm{~min}$, is shown in Figure 2. In these preparations, guanidine induced a gradual increase in the neuromuscular activity, which peak reached $65 \%$ of increase at $20 \mathrm{~min}$; thereafter, there was facilitation reduction, although it has remained approximately $20 \%$ above baseline at the end of $30 \mathrm{~min}$. Removal of guanidine and replacement for Tyrode caused immediate facilitation in a proportion of 95\% (almost twice the initial, primary, facilitation).

Figure 2. Effect of guanidine $(10 \mathrm{mM})$ (white circles) for $30 \mathrm{~min}$ on the twitch response of the PND (A), EDL (C) and SOL (E) $(n=8 /$ each) in comparison with matched controls (black circles) ( $\mathrm{n}=3,8,8$, respectively). The line breaking corresponds to the preparation washing $(\mathrm{W})$. Each point represents the mean \pm S.E.M; one-way ANOVA followed by Tukey test, ${ }^{*} P \leq 0.05$. Myographic isometric twitch records of PND (B), EDL (D) and SOL (F) incubated with guanidine (G). The vertical bars $(5,0.5 \mathrm{~g})$ correspond to muscle tension.

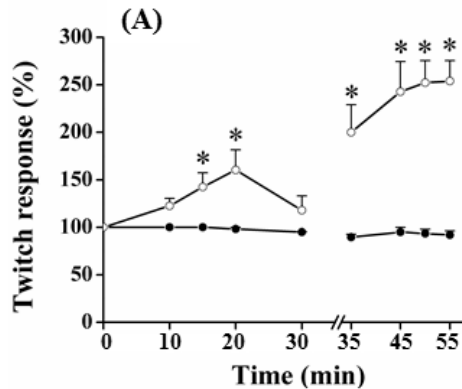

(C)

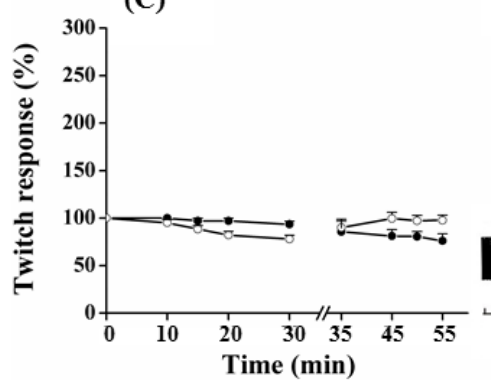

(E)

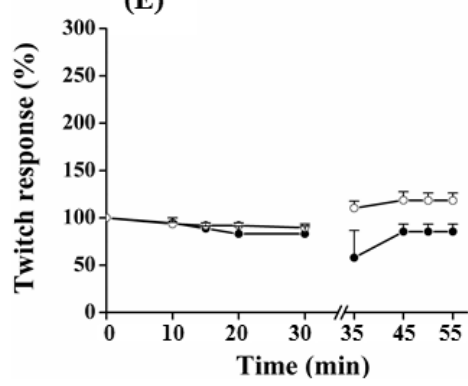

(B)

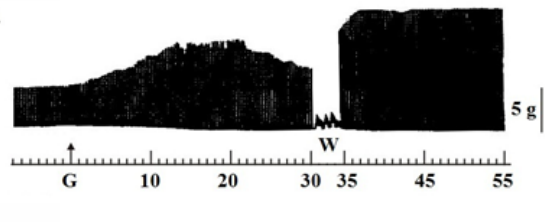

(D)

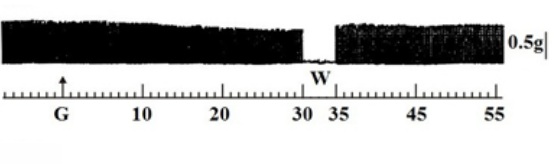

(F)

The amplitude of the contractile response increased during the next $15 \mathrm{~min}$, after which it remained stable; however, it was approximately $150 \%$ superior when comparing with baseline values (Figure 2A,B) $(P \leq 0.05)$. Guanidine $(30 \mathrm{~min})$ to the EDL preparation (Figure 2C,D) depressed not significantly the muscle contractile activity, whereas it caused no change to SOL (Figure 2E,F); the posterior tendency towards a secondary facilitation after guanidine removal from bath was not statistically significant. Neither $1 \mathrm{mM}$ nor $5 \mathrm{mM}$ altered the EDL contractile amplitude (not shown). 
The triphasic effect (primary facilitation/blockade/secondary facilitation of neurotransmission) of guanidine on motor response was selective for PND preparation (Figure 3). A gradual increase in the contraction amplitude was carried out, which peak (45-50\% increase) occurred between 15 and $20 \mathrm{~min}$. In sequence, it gradually decreased leading to total blockade within approximately $40 \mathrm{~min}$ and reaching negative values, approximately $70 \%$ under the baseline value, at $60 \min (P \leq 0.05)$.

Figure 3. Effect of guanidine $(10 \mathrm{mM})$ (white circles) for $60 \mathrm{~min}$ on twitch responses of PND (A), EDL (C) and SOL (E) $(n=8 /$ each) in comparison with matched controls (black circles) ( $\mathrm{n}=3,8,8$, respectively). The line breaking corresponds to the preparation washing (W). Each point represents the mean \pm S.E.M; one-way ANOVA followed by Tukey test, * $P \leq 0.05$. Myographic isometric twitch records of PND (B), EDL (D) and SOL (F) incubated with guanidine $(\mathrm{G})$. The vertical bars $(5,0.5 \mathrm{~g})$ correspond to muscle tension.

(A)
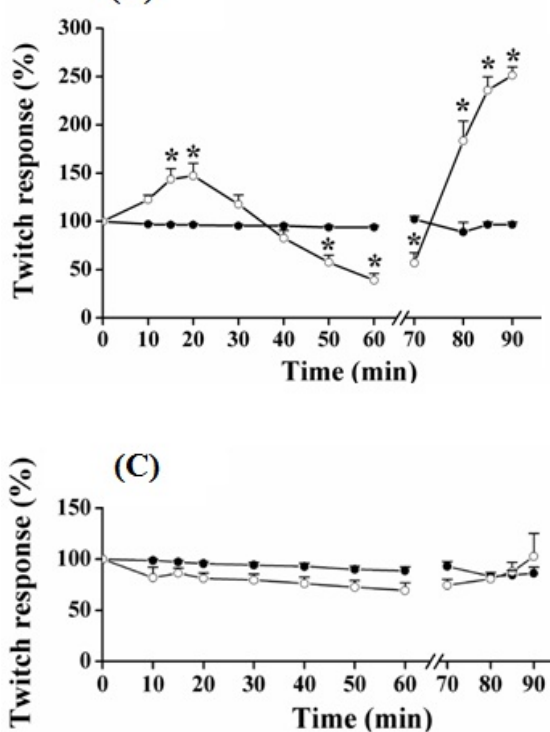

(E)

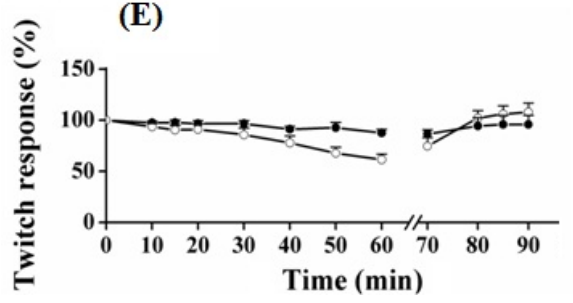

(B)

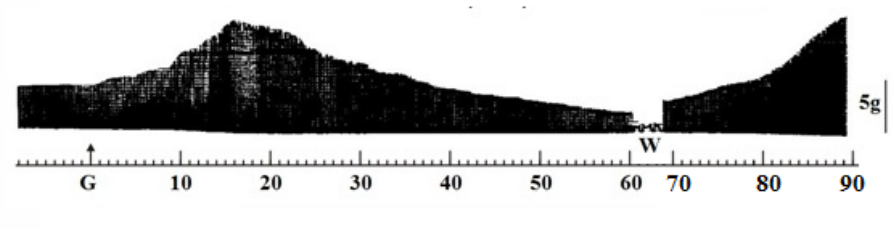

(D)

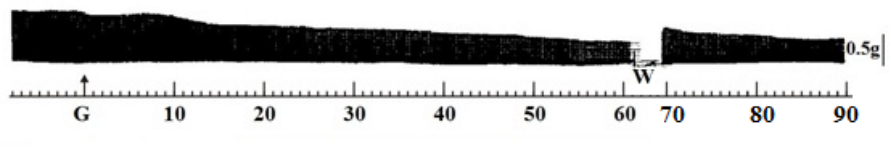

(F)

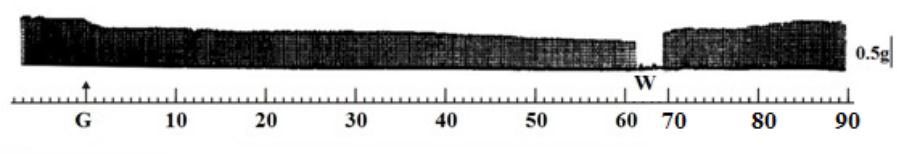

In the diaphragm, the guanidine washout from bathing medium promoted a gradual reversal of neuromuscular blockade, reaching $\sim 150 \%$ of facilitation (secondary) above baseline at $20 \mathrm{~min}$ (Figure 3A,B) $(P \leq 0.05)$. The secondary facilitation can be explained by the ability of guanidine to cross the $\mathrm{Na}^{+}$channel [30,31], and this reduced the miniature endplate potentials (MEPPs) after washing preparations prior incubation with TTX [21]. Neither the primary nor the secondary facilitation was observed in EDL (Figure 3C,D) and SOL (Figure 3E,F) preparations incubated with guanidine for $60 \mathrm{~min}$. The absence of primary and second facilitations in EDL $(10 \mathrm{mM}, 5 \mathrm{mM}$ or $1 \mathrm{mM}$, see Figures 1-4) and SOL (10 mM, Figures 1-3) indicates that the triphasic effect of guanidine on neurotransmission occurs by a complex mechanism of action, depending on intrinsic muscle phenotypic and molecular characteristics. 
Figure 4. Effect of $1 \mathrm{mM}(\mathrm{A})$ and $5 \mathrm{mM}(\mathrm{B})$ guanidine (G) for $60 \mathrm{~min}$ on the twitch response of EDL $(n=3 /$ each). The line breaking corresponds to the preparation washing $(\mathrm{W})$. Each point represents the mean \pm S.E.M; one-way ANOVA followed by Tukey test, * $P \leq 0.05$. Panel B displays the myographic isometric twitch record of these preparations. The vertical bar $(1 \mathrm{~g})$ represents muscle tension.

\section{(A)}

(B)
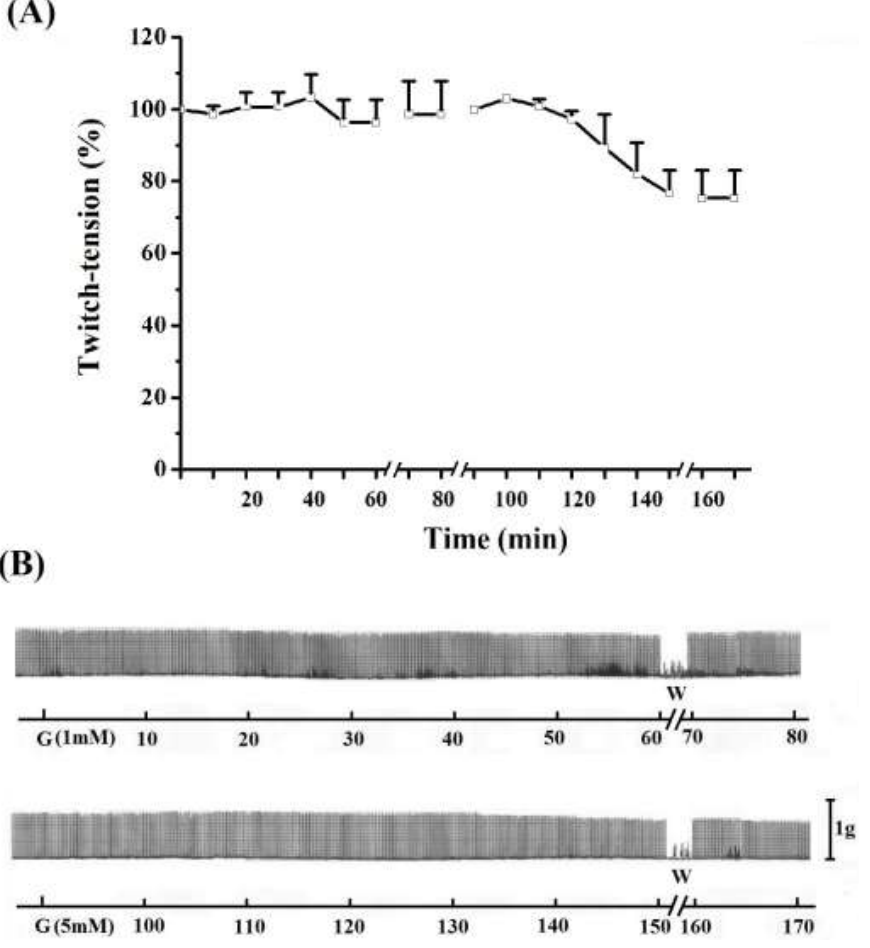

In addition, the effect of $5 \mathrm{mM}$ guanidine was investigated by pre- or post-incubation with 4-aminopyridine (4-AP) in order to check either PND response before or after the blockade of potassium channels. Incubation of PND with 4-AP $(10 \mu \mathrm{g} / \mathrm{mL})$ produced as expected, a rise in the twitch response. The removal of 4-AP from bath by washing returned the twitch amplitude to baseline level. The subsequent addition of $5 \mathrm{mM}$ guanidine produced facilitation of neurotransmission. After 80 min incubation with this low concentration of guanidine there was no blockade or inhibition of the amplitude of twitch response as was usual when $10 \mathrm{mM}$ was used. Instead, the facilitation was maintained. After another washing for guanidine removal from bath the secondary facilitation did occur, almost achieving the level promoted by 4-AP (figure not shown). It was of note that there is a delay (which was variable in time) for starting the neurotransmission facilitation induced by guanidine after the 4-AP incubation and first washing.

We also incubated PND with $5 \mathrm{mM}$ guanidine, then the preparation was washed and 4-AP was added. The addition of guanidine promoted a marked primary facilitation whose amplitude was reduced after 70-80 min. The subsequent washing and addition of 4-AP promoted another facilitation of neurotransmission which was maintained for the subsequent $60 \mathrm{~min}$. The removal of 4-AP from bath by washing the preparation (three times) promoted an almost immediate decrease of the amplitude of the muscle contraction (Figure 5, upper register). A variation of these experiments was done which consisted in incubation of PND with $5 \mathrm{mM}$ guanidine and after $45 \mathrm{~min}$ addition of 4-AP $(10 \mu \mathrm{g} / \mathrm{mL})$ without pre-washing. The 4-AP promoted an immediate rise in the twitch amplitude $(\sim 20 \%)$ which 
persisted until preparation was washed. The washing promoted a secondary facilitation and a third facilitation after the second washing of the preparation. The findings indicate that since guanidine was internalized into the fiber (by traversing sodium channels), the 4-AP pre-treatment did not interfere with the guanidine typical effect in diaphragm preparation (Figure 5, lower register).

Figure 5. Representative recordings (of four experiments each) from indirectly stimulated PND incubated with $5 \mathrm{mM}$ guanidine, followed by washing (w) and subsequent addition of 4-aminopiridinte (4-AP) and another washing after $60 \mathrm{~min}$ additional incubation (upper recording); in the lower recording there was no guanidine removal by washing before 4-AP addition.
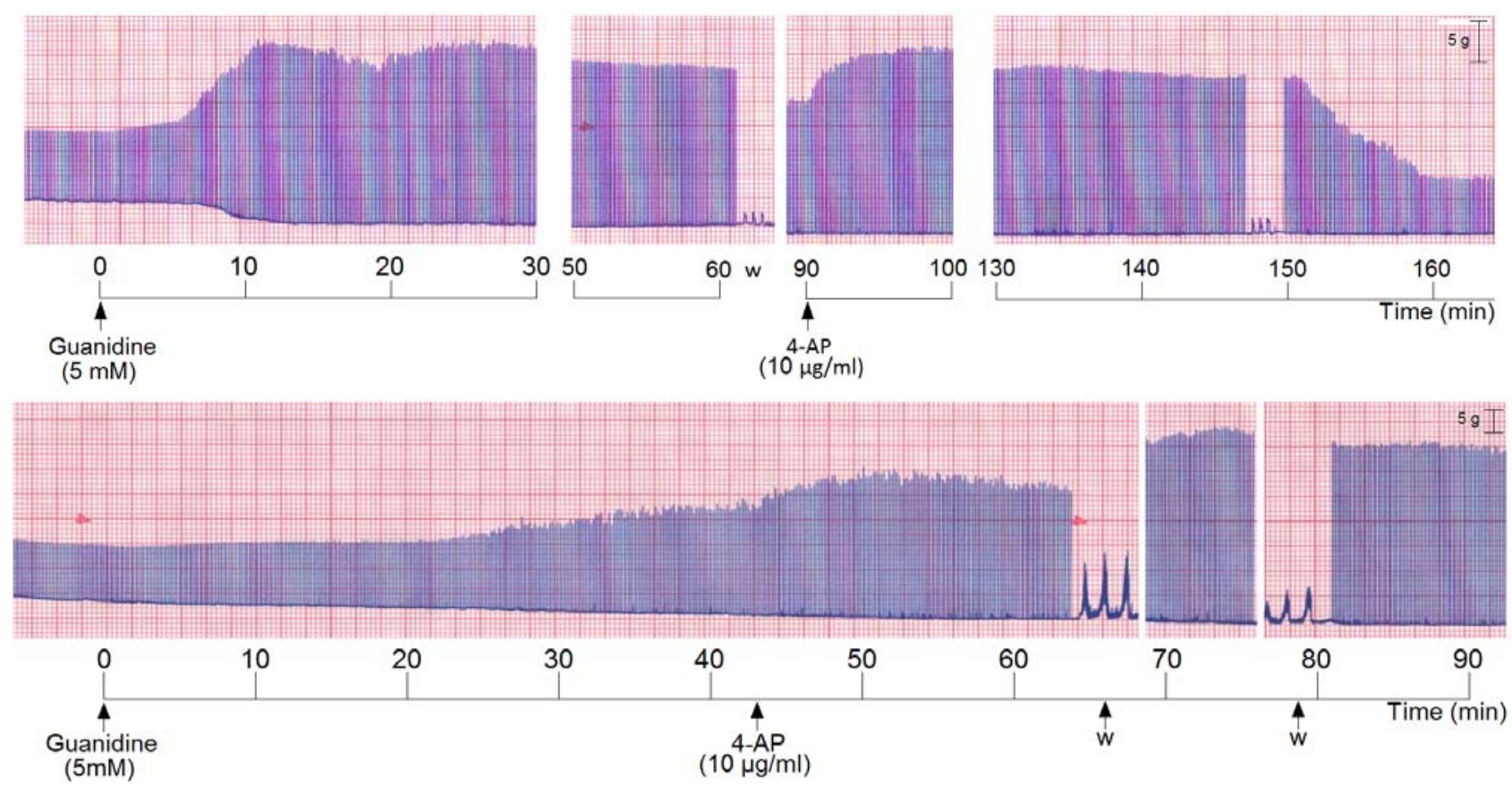

Taken together, the findings show that the blockade of $\mathrm{K}^{+}$channels by 4-AP did not prevent the triphasisc effect characteristic of guanidine. In other words, 4-AP does not interfere with the primary and secondary increase of ACh release by guanidine (even with half of the concentration previously used). The findings suggest that even though both the 4-AP and guanidine are potassium channel blockers, guanidine exhibits some peculiar mechanisms not found in 4-AP. Interestingly when 4-AP is added without previous removal of the guanidine from preparation, there occurs two other facilitatory effects, after 4-AP removal by washing, in addition to the primary one.

Although several authors have suggested that guanidine effect relies on its action on voltage-gated $\mathrm{Na}^{+}, \mathrm{K}^{+}$and $\mathrm{Ca}^{2+}$ channels, present either in the nerve terminal or skeletal muscle, its mechanism of action remains still not totally elucidated [11,21,22,30,32-37]. The time-dependent facilitatory effect of guanidine seen here in the diaphragm could be probably related to presynaptic action of the drug, increasing $\mathrm{Ca}^{2+}$ influx and enhancing release of $\mathrm{ACh}$ by nerve impulse [21,32], consequent to the blockade of $\mathrm{K}^{+}$conductance in the nerve terminal, as well as increasing the duration of the presynaptic action potential [32-36].

The type of stimulation (indirect or direct field stimulation) and/or the guanidine concentration were/was responsible for differences in contractile responses between PND and EDL/SOL 
preparations seeming unlikely. PND even with direct stimulation responded with the typical triphasic effect (data not shown), and incubation with low or high doses of guanidine (5 mM, 20 or $25 \mathrm{mM})$ did not cause the triphasic effect on EDL and SOL preparations even after $60 \mathrm{~min}$ (data not shown).

\subsection{Morphology and Morphometry}

Figure 6 illustrates the normal aspect of muscle fibers of diaphragm incubated with Tyrode (panels A,B) and their different pathological stages in SOL (panel C), EDL (panel D) and diaphragm (panel E) after incubation with $10 \mathrm{mM}$ guanidine for $60 \mathrm{~min}$. Guanidine major effect was the appearance of vacuolated cells in SOL and EDL, which could be due to SR cisternae swelling, as reported for diaphragm [22].

Figure 6. Light micrographs of PND incubated with Tyrode show normal fibers $(\mathrm{N})$ with their well-positioned nuclei (n) (A: longitudinal section, B: transversal section) and of SOL (C), EDL (D) and PND (E) showing different stages of cellular disorganization after incubation with $10 \mathrm{mM}$ guanidine for $60 \mathrm{~min}$, under indirect electrical stimulation; altered cells show vacuolation (V), worm-like threads of myofibrils interspersed among emptylooking regions of sarcoplasm due to sarcolemma disruption (arrows) or hypercontraction caused by densely packed myofibrils (*). See also edematous (round in shape) and non-homogeneously stained myofibers (toluidine blue). Bar: $35 \mu \mathrm{m}$.

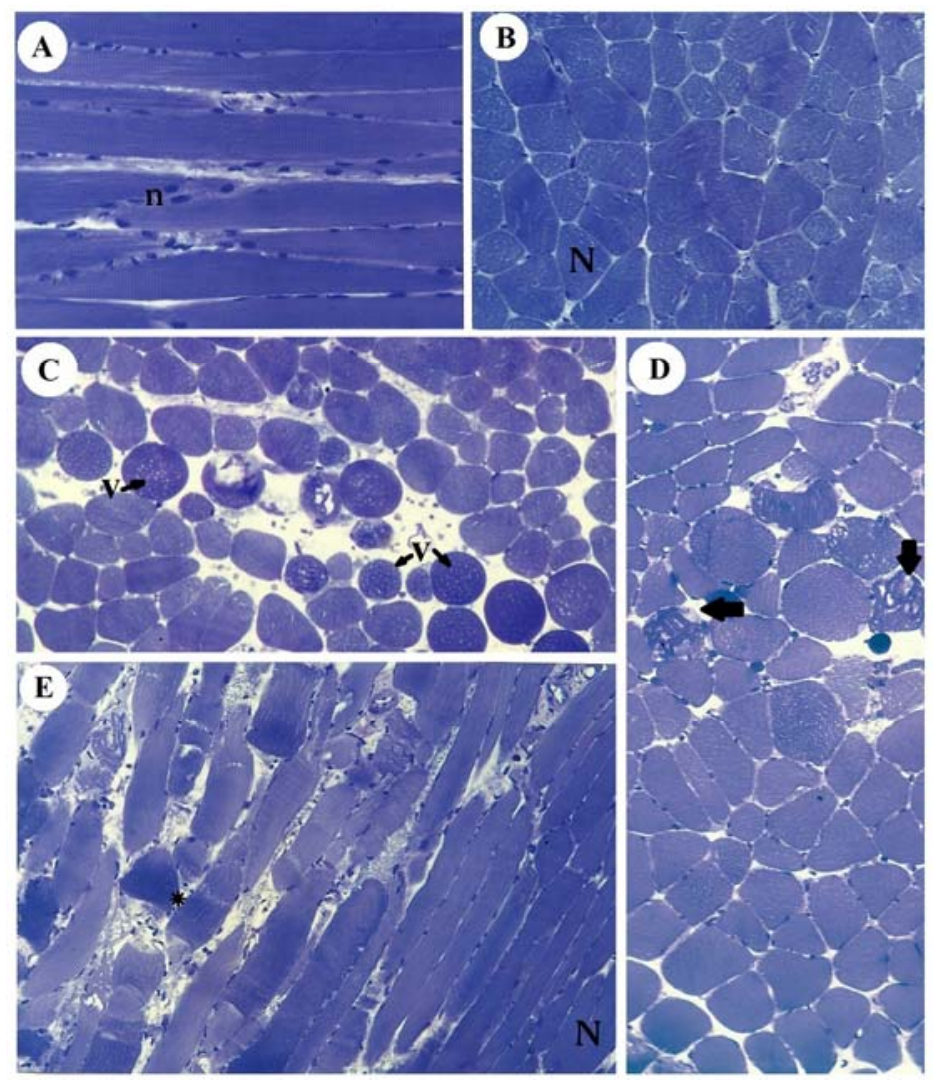

Moreover, instead of a typical polygonal profile in transverse sections, a number of fibers in all three preparations incubated with the organic cation guanidine showed spherical outline characteristic of swollen fibers; only a relative minor number of myofibers presented advanced pathological stages 
with color change due to condensed tortuous bands of myofibrils, or with clear spaces indicative of myofilament loss and sarcolemma rupture. The proportion of affected fibers caused by guanidine and Tyrode incubation is illustrated in Figure 7.

Figure 7. Proportion of affected cells in PND, EDL and SOL muscles after 15, 30 and $60 \mathrm{~min}$ of $10 \mathrm{mM}$ guanidine incubation or Tyrode solution (control). One-way analysis of variance (ANOVA) followed by Tukey test, $* P \leq 0.05$.

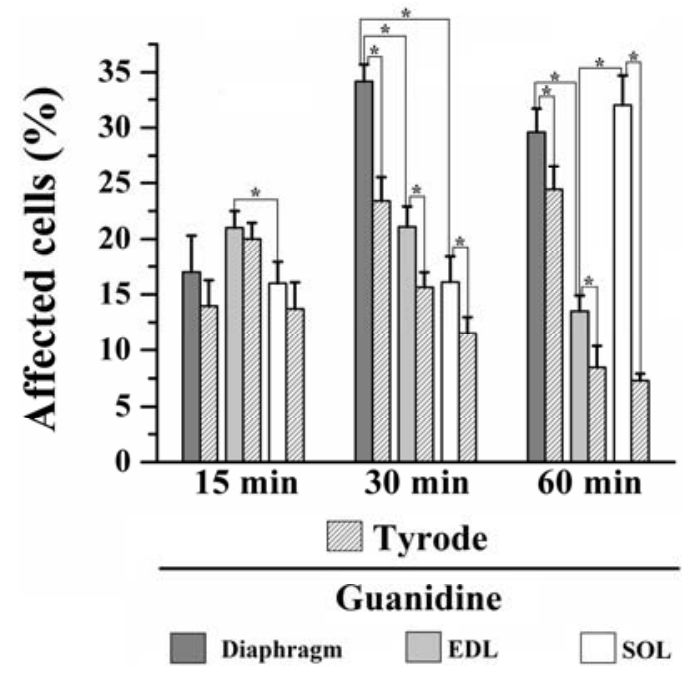

15 minutes incubation: relative to Tyrode-incubated preparations, guanidine did not induce morphological alterations in PND, EDL or SOL (13\% vs. 17\% for PND; $20 \%$ vs. $21 \%$ for EDL and $13 \%$ vs. $16 \%$ for SOL of affected fibers). However, guanidine affected significantly more the EDL than SOL when compared to each other $(P \leq 0.05)$.

30 minutes incubation: relative to control, guanidine promoted a significant increase in the number of affected fibers in all three muscles compared to paired-controls (23\% vs. 33\% for PND; $15 \%$ vs. $21 \%$ for EDL and $11 \%$ vs. $16 \%$ for SOL), $(P \leq 0.05)$. In addition, there was significant difference when comparing the impact of guanidine effect in all three muscles which was highest for PND and lowest for SOL $(P \leq 0.05)$.

60 min incubation: compared to control, guanidine promoted a significant increase in the proportion of fibers damaged in all three muscles (24\% vs. 28\% for PND, $8 \% v s .13 \%$ for EDL and $7.5 \%$ vs. $31 \%$ for SOL $),(P \leq 0.05)$. After $60 \mathrm{~min}, \mathrm{PND}$ and SOL showed higher proportion of damage than EDL $(\mathrm{P} \leq 0.05)$.

The findings give evidences that in vitro incubation with Tyrode solution also promoted fiber morphological alterations. Interestingly, for PND the higher the period of incubation (15, 30 and $60 \mathrm{~min})$ the higher the proportions of fibers affected (13\%, 23\% and $24 \%$, respectively), whilst for EDL and SOL the higher the time of incubation the lower proportion of fibers affected $(20 \%, 15 \%$ and $8 \%$ for EDL and $13 \%, 11 \%$ and $7.5 \%$ for SOL). Taken the findings together, it seems that the interaction of in vitro condition and guanidine effect may give the relative injury caused by guanidine itself. The changes in the proportion of fiber damage may result, at least partially, from a direct action of guanidine on muscle fibers, but also from artifact changes in morphology which can be produced even under satisfactory gassing incubation conditions resulting in cell injury. Curiously, the inverse relation 
of fibers affected versus time duration of Tyrode incubation seen in EDL and SOL suggests that changes were transitory and likely did not involve severe structural alterations in fibers. Based on the findings, the effect of guanidine in EDL may be related with high number of sodium channels in this muscle which interference by the drug provokes alteration of ionic conductance and osmotic disturbances in muscle fibers. This interpretation is in line with the regression in the proportion of fibers affected which passed from $21 \%$ after $15 / 30 \mathrm{~min}$ to $13 \%$ at the end of $60 \mathrm{mi}$. On the other hand, in SOL guanidine effect may involve mitochondrial effect (see below).

The antagonistic effect of guanidine on dantrolene effect [38], a substance able to block the entry of $\mathrm{Ca}^{2+}$ into SR, as well as the secondary facilitation (evoked even under direct stimulation), supports the idea that guanidine has a muscular action, what was currently corroborated. The increase of cytosolic $\mathrm{Ca}^{2+}$ concentration (after $\mathrm{K}^{+}$channel blockade) inside muscle fibers in response to membrane depolarization triggers neurotransmitter release and muscle contraction; the increase in intracellular $\mathrm{Na}^{+}$results in the interruption of the change between extracellular sodium and intracellular calcium. As a result, the high intracellular $\mathrm{Ca}^{2+}$ concentration activates intracellular calcium-dependent proteases, which hydrolyze the components of muscle cells [22,39]. Therefore, the data indicate that, in addition to presynaptic pharmacological effects, guanidine also produces a muscle pharmacological action on the diaphragm $[22,40]$, EDL and SOL.

Molecular characteristics of skeletal muscle types are herein considered as an attempt to explain the differences in the contractile response of PND, EDL and SOL. As premise, it is common sense that the preservation of the membrane electrochemical gradients for $\mathrm{Na}^{+}, \mathrm{K}^{+}$, and $\mathrm{Ca}^{2+}$ is vital to the muscle processes of EC coupling, regardless the muscle phenotype. Herein, we found that evoked end-plate potentials produced a membrane depolarization of diaphragm which was greater in the post-washing secondary facilitation than in the former. This peculiar effect did not find similarity to fast- and slow-twitch muscles. Why EDL and SOL do not respond that way; what are the possible causes of this?

Studies have shown that density and types of ion channels involved in neurotransmission may differ in different muscle fiber types. For instance, NMJ of fast-contracting fibers (glycolytic) have high density of $\mathrm{Na}^{+}$channels, and this density is determined by the type of motor innervation [41-43]. Based on this, it was expected that PND and EDL muscles would present a higher density of $\mathrm{Na}^{+}$ channels than SOL, since they have a higher proportion of glycolytic fibers with fast contraction. As a result, PND and EDL are expected to present a larger proportion of damaged fibers than SOL muscles, which actually occurred in preparations incubated for 15 and $30 \mathrm{~min}$, but not in those incubated for 60 min. Guanidine is able to cross $\mathrm{Na}^{+}$channels [37,38], being prevented from returning to the extracellular compartment by the selectivity of the $\mathrm{Na}^{+}$pump, which does not recognize it [44]. The accumulation of $\mathrm{Na}^{+}$and $\mathrm{Ca}^{2+}$ into the nerve terminal, along with the guanidinium cation influx, is expected to promote osmotic fluid entry and activation of calcium-dependent endoproteases.

Likewise, the existence of different types/proportion of $\mathrm{K}^{+}$channels in all three muscles could have a role in their response to guanidine. $\mathrm{K}^{+}$channels are membrane integral proteins with different types of pore-forming $\alpha$-subunits; they are present ubiquitously in several types of cells where they play key roles in cell function and homeostasis; in skeletal muscle, they maintain the resting membrane potential, regulate the action potential duration and neurotransmitter release $[45,46]$. Recent studies provided evidences that ATP-regulated potassium $\left(\mathrm{K}_{\text {ATP }}^{+}\right)$channel densities and channel subunit composition vary in different muscle fiber types, contributing to their differential physiological 
performance and determining differential pharmacological actions of drugs modulating the channel activity $[47,48] . \mathrm{K}_{\mathrm{ATP}}$ channels were shown to have muscle-specific roles and are physiologically and pharmacologically regulated. They determine muscle phenotype-specific pharmacological control of electrical activity, being its density higher in fast-twitch than in slow-twitch muscles; in so doing, $\mathrm{K}_{\mathrm{ATP}}$ channels play important roles in the control of contractility, protecting tissue against calcium overload (see Flagg et al. [48] for review). Similarly, functional and pharmacological properties of calciumactivated $\mathrm{K}^{+}$channels in skeletal muscle tissue were shown to be also dependent on muscle phenotypic characteristics [49].

The contractile and relaxing activity (EC coupling) of muscle fibers is crucially dependent on the calcium signaling and handling apparatus; as part of the process, there are myriads of regulatory proteins, such as the main proteins (ryanodine receptor, the $\mathrm{SR} \mathrm{Ca}^{2+}$ release channel; troponin, which mediates contraction through the action on $\mathrm{Ca}^{2+}$ interaction with myofibrils; $\mathrm{Ca}^{2+}$ pump, responsible for reuptaking $\mathrm{Ca}^{2+}$ into the $\mathrm{SR}$, and calsequestrin, the $\mathrm{Ca}^{2+}$ storage protein in the SR) and accessory proteins (annexins, beta-actinin, calcineurin, calmodulin, calpain, myosin light chains, parvalbumin, S100 and sorcin), all of them involved in muscle response following a nerve impulse and action potential propagation. Since a range of heavy myosin isoforms may constitute muscle fiber types, the contractile properties of them vary according to their constitutive regulatory and accessory protein modulation [50,51]; thereby a series of $\mathrm{Ca}^{2+}$-dependent physiologic processes will be differentially modulated, including transmitter release in motor nerve endings and contraction in metabolic/physiologic distinct muscle types [27]. For instance, Zubrzycka-Gaarn et al. [51,52] found that the protein amount of $\mathrm{Ca}^{2+}$-ATPase and calsequestrin, both responsible for transporting $\mathrm{Ca}^{2+}$ back into the sarcoplasmic reticulum (SR), was several times lower in the slow-twitch muscle SR vesicles, and that the active $\mathrm{Ca}^{2+}$ transport and formation of the phosphorylated intermediate were several times lower than that observed in fast-twitch muscles. The authors suggested that the slow rate of calcium transport, found in slow-twitch muscle SR vesicles, may be related to a low content of $\mathrm{Ca}^{2+}$-transporting ATPase in the membrane. In addition, the impact of aging on the $\mathrm{Ca}^{2+}$ pump function of skeletal muscle SR, differing in soleus and gastrocnemius of rats, indicates that muscle contractile properties and SR function are muscle specific [52].

It is now established that the regulation of the EC coupling mechanism differs between fast-twitch and slow-twitch fibers and that there is a direct involvement of calsequestrin in the $\mathrm{Ca}^{2+}$ release process, given that protein represents an endogenous modulator of ryanodine receptors $\left(\mathrm{SR} \mathrm{Ca}^{2+}\right.$ release channels) [53,54]. Evidences have been provided that key membrane proteins involved in $\mathrm{Ca}^{2+}$ homeostasis detected fiber-type-specific differences in auxiliary subunits of dihydropyridine receptor, ryanodine receptor and $\mathrm{Ca}^{2+}$-ATPase, as well as in triad markers and various $\mathrm{Ca}^{2+}$-binding and ion-regulatory proteins [55]. The authors concluded that the type of motor innervation has a profound impact on the levels and isoform expression pattern of $\mathrm{Ca}^{2+-}$ regulatory membrane proteins and reflects differences in the regulation of $\mathrm{Ca}^{2+}$ homeostasis and contractile response in different types of muscle.

Feldmeyer et al. [56] suggested that the inhibitory action of guanidinium ion on excitationcontraction coupling is due to a depression of calcium release from the SR. It is possible that the differential contractile response in all three studied muscles is also related to the population of mitochondria present in each type of muscle fiber. Since $\mathrm{Ca}^{2+}$ is one of the main protagonists in the genesis of the triphasic effect of guanidine, and relating it to the fact that mitochondria and SR are the 
main organelles involved in intracellular $\mathrm{Ca}^{2+}$ homeostasis, it is expected that muscle fibers with a larger number of mitochondria are the most affected by guanidine [57]. In fact, SOL muscle, which increased the number of affected cells only after $60 \mathrm{~min}$ of incubation (by more than twice compared to that observed at 15 and $30 \mathrm{~min}$ ) is predominantly constituted of oxidative fibers with a larger proportion of mitochondria. It is known that mitochondria are the main organelles involved in alterations provoked by guanidine, in addition to the participation of the SR in pathophysiological processes of the drug [22,57].

Whether the amount of cells altered by the guanidine action was responsible for such differences is unclear. What is known is that the physiological characteristics of diaphragm [34-37] distinguish this muscle from the peripheral ones, responding differentially to endogenous or exogenous pharmacological stimuli.

The primary and secondary facilitations caused by guanidine specifically for PND suggest that the stimulation threshold for PND to produce a maximum depolarization of the end-plate is not the same stimulation threshold for EDL and SOL muscles, i.e., the threshold to cause an end-plate potential greater for PND is likely smaller than the stimulation threshold for EDL and SOL muscles. What mechanisms can account for such differences? Since diaphragm is a vital muscle, it has the ability to support adverse conditions that peripheral muscles do not have. The term "safety factor" (SF) at the NMJ is used to indicate a property of the diaphragm that maintains neuromuscular transmission operative in conditions not supported by non-vital muscles. The SF relies on both the amount of presynaptic transmitter released per nerve impulse, being greater than that required to trigger an action potential in the muscle fiber, and on elaborate postsynaptic specializations to enhance the transmitter response [58,59]. It also relies on the diaphragm, possessing a higher density of nAChR and ACh quantal contents than peripheral muscles [23]. This means that the SF makes the diaphragm more capable of amplifying the responses to depolarization, promoting the transduction process that leads to contraction than EDL and SOL muscles. Interestingly, Ermilov et al. [60] found that SF varies for neuromuscular transmission across muscle fiber types (even within a single muscle), being larger for type IIX or IIB fibers (fast-twitch types) than for type I or IIA fibers (slow-twitch types). As known, SOL muscle has high predominance of fiber I and IIA types, whereas diaphragm and EDL have high predominance of fiber IIX and IIB types.

A growing interest has been directed towards the mechanism of action of guanidine and its alkyl analogs on potassium channels [20,61]. The increase of ACh release into the synaptic cleft and stimulation of neuromuscular transmission resulting from this action has profound implication in the prospect of drugs targeting neuromuscular diseases. A recent study has shown that guanidine and derivatives bind within the intracellular pore of potassium channel and disturb the hydrophobic subunit interface to stabilize a closed state of the channel [61].

\section{Conclusions}

Guanidine evoked a differential contractile response from diaphragm, extensor digitorum longus and soleus preparations. It seems unrelated to guanidine concentration, stimulation via (direct or indirect) and at some instance to the number of cells intoxicated by the drug. Instead it seems more related to intrinsic nerve-muscle mechanisms, which entail differential interaction of each muscle with 
the drug. Our results point two interesting issues. One issue deals with the post-blockade secondary facilitation of PND after replacement of guanidine for Tyrode solution in the incubation medium. The so-called triphasic effect, selective for PND preparation, appears to be ought to the safe factor (SF) across the NMJ that provides an over presynaptic release of ACh per nerve impulse, being greater than that required to trigger an action potential in the muscle fiber; in addition, the SF would be postsynaptically represented by high proportion of $\mathrm{nAChR}$ present on elaborate junctional fold specializations, typical of diaphragm. Altogether, a lower stimulation threshold would be required for PND to produce a maximum depolarization of the end-plate. A second issue is represented by the unique guanidine action on diaphragm, enhancing neurotransmission, which does not find a similar action to a slow-twitch, predominantly oxidative muscle (SOL), or fast-twitch, predominantly glycolytic muscle (EDL). Considering that the skeletal muscles are composed of different fiber types with heterogeneous density and types of ion channels, mitochondrial population, SR development and metabolic activity, it is suggested that differences in the amplitude of muscle contraction here seen in response to guanidine rely on the fine tune among ion channels versus variable expression of proteins involved in calcium signaling and handling. The present results reiterate the singularity of diaphragm, the largest and most important inspiratory muscle, in its response to pharmacological drugs, so wellendowed of force reserve to support the energy spent in ventilation work. This experimental design allowed imply that guanidine effect on limb and ventilator muscles differ in magnitude, depending on the muscle phenotype and duration of incubation bath. Future studies aimed at investigating the density and types of $\mathrm{K}^{+}, \mathrm{Na}^{+}$and $\mathrm{Ca}^{2+}$ channels and the modulation of the EC coupling machinery associated with SR calcium release/reuptake cycle, mitochondrial function; and/or alterations in the action potential configuration, MEPP and quantal content, being available for the next ACh release in diaphragm, EDL and SOL can shed light on differences relative to interaction with guanidine. The present study formulates several questions to which there are no clear-cut replies; hence, the study opens a vast perspective for investigation that, when responded, will contribute relevantly to the knowledge of NMJ physiology.

\section{Experimental}

\subsection{Animals}

Adult male Swiss mice (Mus musculus) of 30-40 g body weight were supplied by the Multidisciplinary Center for Biological Investigation (Cemib/Unicamp, Campinas, SP, Brazil). The animals were housed at $24{ }^{\circ} \mathrm{C}$ with free access to food (Labina, Purina, Campinas, SP, Brazil) and tap water. The experiments were done in accordance with the guidelines established by the Brazilian Society for Laboratory Animal Science (SBCAL, formerly COBEA) and approved by the University's Ethics Committee on Experimental Animal Use (CEUA/IB/Unicamp, protocol n. 1898-1).

\subsection{Nutritive Solution}

Tyrode solution was used as a nutritive medium for muscle dissection and to provide a physiological fluid for assays on isolated preparations; the solution had the following composition 
[mM]: $\mathrm{NaCl} 137, \mathrm{KCl} 2.7, \mathrm{CaCl}_{2}$ 1.8, $\mathrm{MgCl}_{2}$ 0.49, $\mathrm{NaHCO}_{3}$ 11.9, $\mathrm{NaH}_{2} \mathrm{PO}_{4}$ 0.42, glucose 11.1. The $\mathrm{pH}$ was adjusted to 7.4 and the temperature was kept constant at $37{ }^{\circ} \mathrm{C}$ during twitch recordings.

\subsection{Guanidine Solution}

Guanidine chloride (organic cation salt, G4505, Sigma, St. Louis, MO, USA) was used at concentrations of 1 and $5 \mathrm{mM}$ for EDL and 5 and $10 \mathrm{mM}$ for all nerve-muscle preparation types evaluated.

\subsection{Experimental Protocol}

The contractile response of PND, EDL and SOL was recorded after 15, 30 and $60 \mathrm{~min}$ of guanidine incubation ( $\mathrm{n}=8$ per time interval) under indirect stimulation, based on previous studies showing that the initial facilitation of the contractile response occurred 15 min after PND incubation, blockade occurred between 30-60 min, and the post-washing facilitation was stable 20 min after guanidine removal from incubation bath [19]. After these time-points, guanidine was removed from the bath and replaced by fresh Tyrode solution (three times), and the recording was reestablished for another approximate $20 \mathrm{~min}$. Control preparations were incubated with Tyrode solution ( $\mathrm{n}=3$ PND, $\mathrm{n}=8 \mathrm{EDL}$ and SOL per time interval) under the conditions used for guanidine. Furthermore, PND preparations were either pre-incubated with 4-aminopiridine $(10 \mu \mathrm{g} / \mathrm{mL})$ and then with $5 \mathrm{mM}$ guanidine, or with $5 \mathrm{mM}$ guanidine followed by 4-AP. Between the treatments, the preparation was washed $(3 \times)$ for removal of the substance before addition of the other. Experiment with and without washing was done exclusively when guanidine was added before 4-AP.

\subsection{Diaphragm Preparation}

After being anesthetized with halothane inhalation (Cristália, Campinas, SP, Brazil), the mice were sacrificed by exsanguination and diaphragms were excised together with the left and right phrenic nerve trunks. The left nerve-hemidiaphragm preparation was mounted according to the method of Bülbring [61] for rat. PND preparation was suspended in a $5 \mathrm{~mL}$ organ bath aerated with carbogen $\left(95 \% \mathrm{O}_{2}\right.$ plus $\left.5 \% \mathrm{CO}_{2}\right)$ and kept under a constant $5 \mathrm{~g} / \mathrm{cm}$ tension in Tyrode solution $\left(\mathrm{pH} 7.4,37^{\circ} \mathrm{C}\right)$. The preparation was indirectly stimulated using electrodes placed around the phrenic nerve $(0.1 \mathrm{~Hz}$, $0.2 \mathrm{~ms}$, supramaximal stimuli until $3 \mathrm{~V}$ ), delivered from a Grass S4 electronic stimulator). Isometric muscle twitch was recorded by a force displacement transducer (Load Cell BG-10 GM, Kulite Semiconductor Products Inc., NJ, USA) coupled to a physiograph (Gould Universal Amplifier, model RS 3400, Cleveland, OH, USA). The preparation was allowed to stabilize at least 20 min before guanidine $(10 \mathrm{mM})$ addition.

\subsection{EDL and SOL Preparations}

Briefly, mouse isolated extensor digitorum longus and soleus muscles were removed under anesthesia. Both muscles were isolated from their distal to proximal tendon insertion, under continuous Tyrode solution bathing. The proximal tendon was fixed to the bottom of the organ bath $(3 \mathrm{~mL}$ of Tyrode solution aerated with carbogen, $95 \% \mathrm{O}_{2}$ plus $5 \% \mathrm{CO}_{2}, \mathrm{pH} 7.4,37^{\circ} \mathrm{C}$, and kept under a constant $0.5 \mathrm{~g} / \mathrm{cm}$ tension) and the distal one was connected to an isometric transducer coupled to a 
physiograph. The preparations were electrically stimulated at the equatorial region of the muscles, using a platinum bipolar electrode. Stimuli were likewise maximal (until 6-8 V, $0.1 \mathrm{~Hz}$ frequency and $0.2 \mathrm{~ms}$ duration). After stabilization (at least $20 \mathrm{~min}$ ), guanidine (10 $\mathrm{mM}$ for SOL and 5 and $10 \mathrm{mM}$ for EDL) was added to the bath.

\subsection{Morphological Analysis and Morphometry}

At the end of the myographic recordings $(15,30$ or $60 \mathrm{~min})$, the muscles were fixed in Bouin's acetic solution for 24-30 hours. Diaphragm tissue samples were collected from the phrenic nerve branching region and consisted of $\sim 5 \times 8 \mathrm{~mm}$ and $5 \times 4 \mathrm{~mm}$ tissue pieces. EDL and SOL muscle samples were obtained from the equatorial region, while the tendon region was discarded. Muscle fragments were dehydrated in increasing ethanol series and processed for embedding in historesin (Leica). Serial sections, $2 \mu \mathrm{m}$ thick, were stained with toluidine blue and mounted in Entelan. The extent of muscle damage was assessed qualitatively and quantitatively (transversal section) by counting 1000 fibers (normal or damaged) in three non-overlapping randomized areas with $80 \mu \mathrm{m}$ of distance from each other per preparation totaling 6000 fibers counted per treatment. Morphometric analyses were done in a BX51 Olympus light microscope (Tokyo, Japan) coupled to an image analyzer and two morphological patterns were established and quantified: 1) normal cells were those which appeared with no morphological abnormality in the myofibrillar organization, nucleus location, and typical polygonal profile; 2) damaged cells were those showing microvacuoles, changes in color (intensely stained), partial or total digestion of myofibrils, presence of delta-shaped lesions or visible interruptions of the fiber membrane.

\subsection{Statistical Analysis}

Each experimental protocol was repeated from three to eight times and the results reported as the mean \pm S.E.M. were used for statistical comparison, using one-way analysis of variance (ANOVA) followed by Tukey's post-hoc test, with a $p$-value $\leq 0.05$ indicating significance.

\section{Acknowledgments}

We thank Mr. Gildo B. Leite for his excellent technical assistance and Mr. Miguel B. Silva for caring for the animals, as well as Chariston A. Dal Belo, from Unipampa University (RS, Brazil), for reading critically the manuscript. R.F. was supported by scholarship from the Coordination for the Improvement of Higher Level Personnel (Capes); M.A.C.H. is I-A level research fellow of the National Council for Scientific and Technological Development (CNPq, Proc. n. 303273/2005-4). The study was supported by the State of São Paulo Research Foundation (FAPESP, grants \# 93/4995-5 and \# 96/11653-8).

\section{Conflict of Interest}

None. 


\section{References}

1. Duchen, L.W.; Gomez, S.; Queiroz, L.S. The neuromuscular junction of the mouse after black widow spider venom. J. Physiol. 1981, 316, 279-291.

2. Rodrigues-Simioni, L.; Borgese, N.; Ceccarelli, B. The effects of Bothrops jararacussu venom and its components on frog nerve-muscle preparation. Neuroscience 1983, 10, 475-489.

3. Cruz-Höfling, M.A.; Love, S.; Brook, G.; Duchen, L.W. Effects of Phoneutria nigriventer spider venom on mouse peripheral nerve. Q. J. Exp. Physiol. 1985, 70, 623-640.

4. Costa, P.D.; Toyama, M.H.; Rodrigues-Simioni, L.; da Cruz-Höfling, M.A. Effects of Bothrops pirajai venom on the mouse externsor digitorum longus (EDL) muscle preparation. Toxicon 1999, 37, 1143-1153.

5. Roed, A. An inhibitory effect of veratridine during tetanic stimulation of the rat diaphragm. Acta Physiol. Scand. 1994, 150, 389-395.

6. Re, L.; Corneli, C.; Sturani, E.; Paolucci, G.; Rossini, F.; Léon, O.S.; Martínez, G.; Bordicchia, M.; Tomassetti, Q. Effects of Hypericum extract on the acetylcholine release: A loose patch clamp approach. Pharmacol. Res. 2003, 48, 55-60.

7. Granjeiro, M.S.; Calheiros-Lima, A.P.; Martins, M.F.; Arruda, L.F.; Garcez-do-Carmo L.; Santos, W.C. Pharmacological effects of Eugenisa punicifolia (Myrtaceae) in cholinergic nicotinic neurotransmission. J. Ethonopharmacol. 2006, 108, 26-30.

8. Stanley, E.F. Presynaptic calcium channels and the transmitter release mechanism. Ann. N.Y. Acad. Sci. 1993, 681, 368-372.

9. Stanley, E.F.; Cox, C. Calcium channels in the presynaptic nerve terminal of the chick ciliary ganglion giant synapse. Ann. NY Acad. Sci. 1991, 635, 70-79.

10. Lindgren, C.A.; Moore, J.W. Calcium current in motor nerve endings of the lizard. Ann. N.Y. Acad. Sci. 1991, 635, 58-69.

11. Lundh, H.; Leander, S.; Thesleff, S. Antagonism of the paralysis produced by botulinum toxin in the rat. The effects os tetraetylammonium, guanidine and 4-aminopyridine. J. Neurol. Sci. 1977, 32, 29-43.

12. Bosch, F.; Morales, M.; Badia, A.; Baños, J.E. Influence of extracellular calcium on the actions of guanidine at the rat neuromuscular junction. Arch. Int. Pharmacodyn. Ther. 1992, 315, 110-119.

13. Molgó, J.; Tabti, N. A review on drugs and toxins affecting presynaptic $\mathrm{K}^{+}$currents and phasic quantal transmitter release at motor nerve terminals. Acta Physiol. Pharmacol. Lationoam. 1989, 39, 333-342.

14. Molgó, J.; Mallart, A. The mode of action of guanidine on mouse motor nerve terminals. Neurosci. Lett. 1988, 89, 161-164.

15. Minot, A.S.; Dodd, K.; Riven, S.S. Use of guanidine hydrochloride in treatment of myasthenia gravis. Am. Med. Assoc. 1939, 113, 553-559.

16. Elmqvist, D.; Lambert, E.H. Detailed analysis of neuromuscular transmission in a patient with the myasthenic syndrome sometimes associated with bronchogenic carcinoma. Mayo Clinic. Proc. 1968, 43, 689-713.

17. Feng, T.P. Studies on the neuromuscular junction. The effects of guanidine. Clin. J. Physiol. 1938, 13, 119-140. 
18. Feng, T.P. Studies on neuromuscular junctions: Local potentials around neuromuscular junctions evoked by single and multiple volleys. Clin. J. Physiol. 1940, 15, 367-404.

19. Doebler, J.A. Gramicidin toxicity in NG108-15 cells: Protective effect of acetamidine and guanidine. Cell Biol. Toxicol. 1999, 15, 279-289.

20. Sorensen, U.S.; Eriksen, B.L.; Teuber, L.; Peters, D.; Strobaek, D.; Johansen, T.H.; Christophersen, P. Novel guanidine derivatives and their Medical Use. WO/2007/110363, 4 October 2007.

21. Rodrigues-Simioni, L.; Silva-Carvalho, I.; Heluany, N.F.; Leite, G.B.; Prado-Franceschi J.; Cruz-Höfling, M.A.; Ballejo, G.; Corrado, A.P. Novel effects of guanidine on the neuromuscular junction. Gen. Pharmacol. 1997, 28, 599-605.

22. Cruz-Höfling, M.A.; Rodrigues-Simioni, L. In vitro studies of the ultrastructural changes induced by guanidine in the nerves, muscle fibers and neuromuscular junction of the mouse diaphragm. Gen. Pharmacol. 1998, 30, 705-711.

23. Nguyen-Huu, T.; Molgo, J.; Servent, D.; Duvaldestin, P. Resistance to d-tubocurarine of the rat diaphragm as compared to a limb muscle influence of quantal transmitter release and nicotinic acetylcholine receptors. Anesthesiology 2009, 110, 1011-1015.

24. Nguyen-Huu, T.; Dobbertin, A.; Barbier, J.; Minic, J.; Krejci, E.; Duvaldestin, P.; Molgó, J. Cholinesterases and the resistance of the mouse diaphragm to the effect of tubocurarine. Anesthesiology 2005, 103, 788-795.

25. Fournier, M.; Sieck, G.C. Mechanical properties of muscle units in the cat diaphragm. J. Neurophysiol. 1988, 59, 1055-1066.

26. Rowley, K.L.; Mantilla, C.B.; Ermilov, L.G.; Sieck, G.C. Synaptic vesicle distribution and release at rat diaphragm neuromuscular junctions. J. Neurophysiol. 2007, 98, 478-487.

27. Schiaffino, S.; Reggiani, C. Fiber types in skeletal muscle. Physiol. Rev. 2011, 91, 1447-1531.

28. Burnstock, G.; Kennedy, C. P2X receptors in health and disease. Adv. Pharmacol. 2011, 61, 333-372.

29. Cain, S.M.; Snutch, T.P. Voltage-gated calcium channels and disease. Biofactors 2011, 37, 197-205.

30. Campbell, D.T. Ionic selectivity of the sodium channel of frog skeletal muscle. J. Gen. Physiol. 1976, 67, 295-307.

31. Dwyer, T.M.; Adams, D.J.; Hille, B. The permeability of the endplate channel to organic cations in frog muscle. J. Gen. Physiol. 1980, 75, 469-492.

32. Mattthews, G.; Wickelgren, W.O. Effects of guanidine on transmitter release and neuronal excitability. J. Physiol. 1977, 266, 69-89.

33 Larramendi, L.M.H.; Lorente Di Nó, R.; Vidal, F. Restoration of sodium-deficient frog nerve fibres by an isotonic solution of guanidinium chloride. Nature 1956, 178, 316-317.

34. Tasaki, I.; Singer, I.; Watanabe, A. Excitation of internally perfused squid giant axons in sodium-free media. Proc. Nat. Acad. Sci. USA 1965, 54, 763-769.

35. Tasaki, I.; Singer, I.; Watanabe, A. Excitation of squid giant in sodium-free external media. J. Physiol. 1966, 211, 746-754.

36. Banks, F.W. The effect of guanidine on transmitter release in the ciliary ganglion of the chick. J. Physiol. 1978, 278, 425-433. 
37. Hille, B. The selective inhibition of delayed potassium currents in nerves by tetraethylammonium. J. Gen. Physiol. 1967, 50, 1287-1302.

38. Silva-Carvalho, I. Contribution to the Study of Guanidine on the Neuromuscular Junction. Doctoral Thesis. Faculty of Dentistry of Piracicaba (FOP), University of Campinas, Piracicaba, SP, Brazil, 1991; p. 86.

39. Melo, P.A.; Ownby, C.L. Different sensitivity of fast- and slow-twitch muscles to some snake venoms and myotoxins. Toxicon 1996, 34, 653-669.

40. Cruz-Höfling, M.A.; Silva-Carvalho, I.; Leite, G.B.; Corrado, A.P.; Rodrigues-Simioni, L. Myonecrosis induced by guanidine in the mouse isolated phrenic nerve diaphragm preparation. Gen. Pharmacol. 1997, 28, 593-597.

41. Ruff, R.L. $\mathrm{Na}^{+}$current density at and away from end plates on fast- and slow-twitch skeletal muscle fibers. Am. J. Physiol. 1992, 262, C229-C234.

42. Milton, R.L.; Behforouz, M.A. $\mathrm{Na}^{+}$channel density in extra junctional sarcolemma of fast and slow twitch mouse skeletal muscle fibres: Functional implications and plasticity after fast motoneuron transplantation to a slow muscle. J. Muscle Res. Cell. Motil. 1995, 16, 430-439.

43. Ruff, R.L.; Whittlesey, D. $\mathrm{Na}^{+}$currents near and away from endplates on human fast and slow twitch muscle fibers. Muscle Nerve 1993, 16, 922-929.

44. Keynes, R.D. Ions channel in the nerve-cell membrane. Sci. Am. 1979, 240, 98-107.

45. Rudy, B. Diversity and ubiquity of K channels. Neuroscience 1988, 25, 729-749.

46. Snyders, D.J. Structure and function of cardiac potassium channels. Cardiovasc. Res. 1999, 42, 377-390.

47. Tricarico, D.; Mele, A.; Lundquist, A.L.; Desai, R.R.; George, A.L., Jr.; Conte Camerino, D. Hybrid assemblies of ATP-sensitive $\mathrm{K}^{+}$channels determine their muscle-type-dependent biophysical and pharmacological properties. Proc. Natl. Acad. Sci. USA 2006, 103, 1118-1123.

48. Flagg, T.P.; Enkvetchakul, D.; Koster, J.C.; Nichols, C.G. Muscle $\mathrm{K}_{\text {ATP }}$ channels: Recent insights to energy sensing and myoprotection. Physiol. Rev. 2010, 90, 799-829.

49. Tricarico, D.; Mele, A.; Conte Camerino, D. Phenotype-dependent functional and pharmacological properties of BK channels in skeletal muscle: Effect of microgravity. Neurobiol. Dis. 2005, 20, 296-302.

50. Pette, D.; Staron, R.S. Myosin isoforms, muscle fiber types, and transitions. Microsc. Res. Tech. 2000, 50, 500-509.

51. Zubrzycka-Gaarn, E.; Korczak, B.; Osinska, H.; Sarzala, M.G. Studies on sarcoplasmic reticulum from slow-twitch muscle. J. Muscle Res. Cell. Motil. 1982, 3, 191-212.

52. Westerblad, H.; Allen, D.G. Changes of myoplasmic calcium concentration during fatigue in single mouse muscle fibers. J. Gen. Physiol. 1991, 98, 615-635.

53. Damiani, E.; Margreth, A.J. Characterization study of the ryanodine receptor and of calsequestrin isoforms of mammalian skeletal muscles in relation to fibre types. Muscle Res. Cell Motil. 1994, $15,86-101$.

54. Murray, B.E.; Ohlendieck, K. Complex formation between calsequestrin and the ryanodine receptor in fast- and slow-twitch rabbit skeletal muscle. Febs. Lett. 1998, 429, 317-322. 
55. Froemming, G.R.; Murray, B.E.; Harmon, S.; Pette, D.; Ohlendieck, K. Comparative analysis of the isoform expression pattern of $\mathrm{Ca}(2+)$-regulatory membrane proteins in fast-twitch, slow-twitch, cardiac, neonatal and chronic low-frequency stimulated muscle fibers. Biochim. Biophys. Acta 2000, 1466, 151-168.

56. Feldmeyer, D.; Csemoch, L.; Kovács, L.; Thieleczek, R. Effects of guanidinium on EC coupling and tension generation in frog skeletal muscle. J. Muscle Res. Cel. Motil. 1988, 9, 541-551.

57. Davidoff, F. Effects of guanidine derivatives on mitochondrial function. J. Biol. Chem. 1974, 249, 6406-6415.

58. Wood, S.J.; Slater, C.R. Safety factor at the neuromuscular junction. Prog. Neurobiol. 2001, 64, 393-429.

59. Ruff, R.L. Neurophysiology of the neuromuscular junction: Overview. Ann. NY Acad. Sci. 2003, 998, 1-10.

60. Ermilov, L.G.; Mantilla, C.B.; Rowley, K.L.; Sieck, G.C. Safety factor for neuromuscular transmission at type-identified diaphragm fibers. Muscle Nerve 2007, 35, 800-803.

61. Kalia, J.; Swatz, K.J. Elucidating the molecular basis of action of a classic drug: Guanidine compounds as inhibitors of voltage-gated potassium channels. Mol. Pharmacol. 2011, 80, $1085-1095$.

Sample Availability: Samples of the compounds Guanidine chloride are available from the authors.

(C) 2012 by the authors; licensee MDPI, Basel, Switzerland. This article is an open access article distributed under the terms and conditions of the Creative Commons Attribution license (http://creativecommons.org/licenses/by/3.0/). 